\title{
Energy Efficient Downlink Transmission in Wireless LANs by Using Low-Power Wake-Up Radio
}

\author{
Suhua Tang and Sadao Obana \\ Graduate School of Informatics and Engineering, The University of Electro-Communications, Chofu, Japan \\ Correspondence should be addressed to Suhua Tang; shtang@uec.ac.jp
}

Received 26 August 2017; Accepted 28 November 2017; Published 24 December 2017

Academic Editor: Zhi Liu

Copyright (C) 2017 Suhua Tang and Sadao Obana. This is an open access article distributed under the Creative Commons Attribution License, which permits unrestricted use, distribution, and reproduction in any medium, provided the original work is properly cited.

\begin{abstract}
In the downlink of a wireless LAN, power-save mode is a typical method to reduce power consumption. However, it usually causes large delay. Recently, remote wake-up control via a low-power wake-up radio (WuR) has been introduced to activate a node to instantly receive packets from an access point (AP). But link quality is not taken into account and protocol overhead of wake-up per node is relatively large. To solve these problems, in this paper, a broadcast-based wake-up control framework is proposed, and a low-power WuR is used to receive traffic indication map from an AP, monitor link quality, and perform carrier sense. Among the nodes which have packets buffered at the AP, only those whose SNR is above a threshold will be activated, contending via a proper contention window to receive packets from the AP. Optimal SNR threshold, deduced by theoretical analysis, helps to reduce transmission collisions and false wake-ups (caused by wake-up latency) and improve transmission rate. Extensive simulations confirm that the proposed method (i) effectively reduces power consumption of nodes compared with other methods, (ii) has less delay than power-save mode in times of light traffic, and (iii) achieves higher throughput than other methods in the saturation state.
\end{abstract}

\section{Introduction}

Wireless local area network (WLAN) [1] is playing a more and more important role in offloading the ever-increasing mobile traffic from cellular networks. Downlink traffic is the majority in WLANs, and its transmission usually faces two problems. (i) First one is the energy waste due to idle waiting. Because the arrival timing of a packet is not known in advance, the WLAN module of each node has to stay awake so as to receive any packet instantly. This constant awake mode leads to much energy waste. Power-save mode [1] (and adaptive PSM [2]) is a typical method to reduce power consumption. In this method, WLAN modules of nodes periodically wake up to receive from the AP (Access Point) beacons with traffic indication, and if any packets have arrived, they stay awake to receive those packets. However, in this duty-cycling mode [3], saving more power requires a longer period, which usually leads to larger delay. (ii) Second one is the low transmission rate or even packet loss due to multipath fading. This is because an AP has no channel state information (CSI) about its nodes.
As for (i), there has been much research on remote wakeup control $[4,5]$. The basic idea is to equip each node with a low-power wake-up radio (WuR), which is always active to accept wake-up request [6-8]. Then, power-hungry parts of a node are put into sleep and remotely activated on demand. This was first studied for sensor networks [9-11]. Because there is little traffic in sensor networks, most existing work adopted a loose integration of the $\mathrm{WuR}$ and the sensor module.

Recently, the idea of wake-up control is also applied to WLAN. In the simple downlink wake-up control [12], an AP transmits a wake-up message (WuM) to a specific node. A WuR receiving a WuM matching its own address activates its colocated WLAN module on demand. However, this unicast wake-up per node generates much overhead, and transmissions may be conducted at a low rate or even packet loss occurs in the presence of multipath fading.

As for (ii), many efforts have been devoted to applying multiuser scheduling to improve transmission rate, and there is still active research in this field $[13,14]$. In the multiple access uplink, many nodes contend to access the shared 
channel to transmit to the AP. The performance depends on two important factors: (i) collision probability and (ii) transmission rate. Because each node experiences fading independently, by limiting the contention to nodes with high link quality, average transmission rate can be improved and collision probability can be reduced. There have been some methods suggested for slotted ALOHA $[15,16]$ and CSMA networks [17]. This is also applied to the downlink transmission by letting each node contend to initiate the receiving from the AP, based on their link quality [18]. But a WLAN module is kept awake to monitor the channel continuously to obtain fresh CSI, which leads to much energy waste.

Compared with previous works that separately solve the above issues, in this work, we try to jointly solve these issues, applying multiuser scheduling to improve transmission rate, meanwhile reducing both the protocol overhead of wake-up control and the energy overhead of monitoring the channel, all by using the low-power WuR. To this end, we propose a new framework, broadcast-based wake-up control. In this framework, an AP periodically broadcasts by its WLAN module a WuM carrying a traffic indication map (TIM) [1], which is modulated by OOK (On-Off-Keying) on the envelope of an OFDM signal [19]. This WuM is received by envelope detection at a low-power WuR equipped at each node. A WuR is used to monitor link quality, performs carrier sense, and activates its colocated WLAN module via the distributed contention.

The broadcast-based wake-up control brings new issues. When more than two nodes contend, there will be transmission collisions and false wake-ups. A WLAN module cannot transmit immediately after it receives wake-up instruction from its $\mathrm{WuR}$ in the presence of the wake-up latency. During the wake-up period of a node, other WuRs sense the channel as idle and falsely activate their WLAN modules, which lead to false wake-up [20] and power waste. The false wakeup problem occurs because the initial values of backoff counters of two or more WuRs have a difference less than the length of the wake-up period. This problem is addressed in [20] by adjusting the contention window, which uses larger contention window at the cost of more idle slots. In this work, this issue is solved by setting the SNR (signal to noise ratio) threshold and applying the distributed scheduling of wake-up control and transmission. This reduces transmission collisions as well.

Main contributions of this paper are threefold as follows:

(1) A new framework of broadcast-based wake-up control enables distributed wake-up scheduling.

(2) Broadcast-based wake-up control reduces protocol overhead and improves channel efficiency compared with wake-up per node.

(3) The three problems, multipath fading, transmission collisions, and false wake-ups, are solved simultaneously, by setting a proper SNR threshold.

Optimal SNR threshold is deduced by theoretical analysis. Extensive simulations verify that the proposed method (i) effectively reduces power consumption of nodes compared with the other methods, (ii) has less delay than powersave mode in times of light traffic, and (iii) achieves higher throughput than the other methods in the saturation state.

The rest of this paper is organized as follows: Section 2 presents the proposed framework, Section 3 gives the optimal parameter by theoretical analysis, Section 4 shows the results of simulation evaluation, and finally Section 5 concludes this paper.

\section{Proposed Method}

The framework of the proposed method is shown in Figure 1. In this framework, a low-power WuR is added to each node for activating its colocated WLAN module. To suppress power consumption, a WuR instead of a power-hungry WLAN module is used to monitor the channel and perform carrier sense. To this end, a WuR shares the same channel with WLAN modules and a WuM is transmitted from the WLAN module of an AP to a WuR.

2.1. Wake-Up Model. Different methods can be used to convey a WuM from a WLAN module (of an AP) to a non-WLAN WuR as follows. (i) Frame length of WLAN signals can be modulated to deliver wake-up message from a WLAN module (transmitter) to a WuR [21-23]. (ii) A WLAN module, at a low clock rate, can work as a WuR to detect a WuM embedded in the preamble of a WLAN signal [24]. (iii) OOK modulation can be emulated to transmit a WuM by modulating the envelope of an OFDM signal [19]. In this work, we adopt (iii) because a WuR with envelope detection can work with low-power consumption, and OOK modulation is more efficient than frame length modulation.

A WuM carried in the envelope of a WLAN signal is detected by a WuR using envelope detection. It should be noted that emulating the OOK modulation has its own cost. For example, at the rate of $100 \mathrm{kbps}$, transmitting a WuM with 40 bits will require $0.4 \mathrm{~ms}$, which causes much overhead for wake-up per node. To reduce protocol overhead, a broadcast wake-up policy is adopted in this work, instead of letting an AP separately activate each node to receive packets. To this end, each WuM carries a TIM, which simultaneously notifies packet availability to multiple nodes. Then, it is nodes, instead of the AP, that contend to initiate the data receiving [25] by transmitting a CTS (clear to send) frame to the AP. This CTS tells the AP that the transmitting node has changed from the sleep mode to the active mode.

Each WuM initiates the transmission of all packets indicated by a WuM. The frame control field in each data frame carries a More Data flag [1], by which the AP notifies a node whether any data remain for that node to receive (to ensure fair scheduling, an AP can clear the More Data flag to a node when the allocated time for that node has run out, although actually there are still packets waiting for transmission to that node). A node without any more data will go to sleep, after notifying the AP.

A WuM has the same preamble as a WLAN signal. Therefore, a WuR can estimate SNR/RSSI of a WuM and on this basis performs carrier sense, contending to activate its 


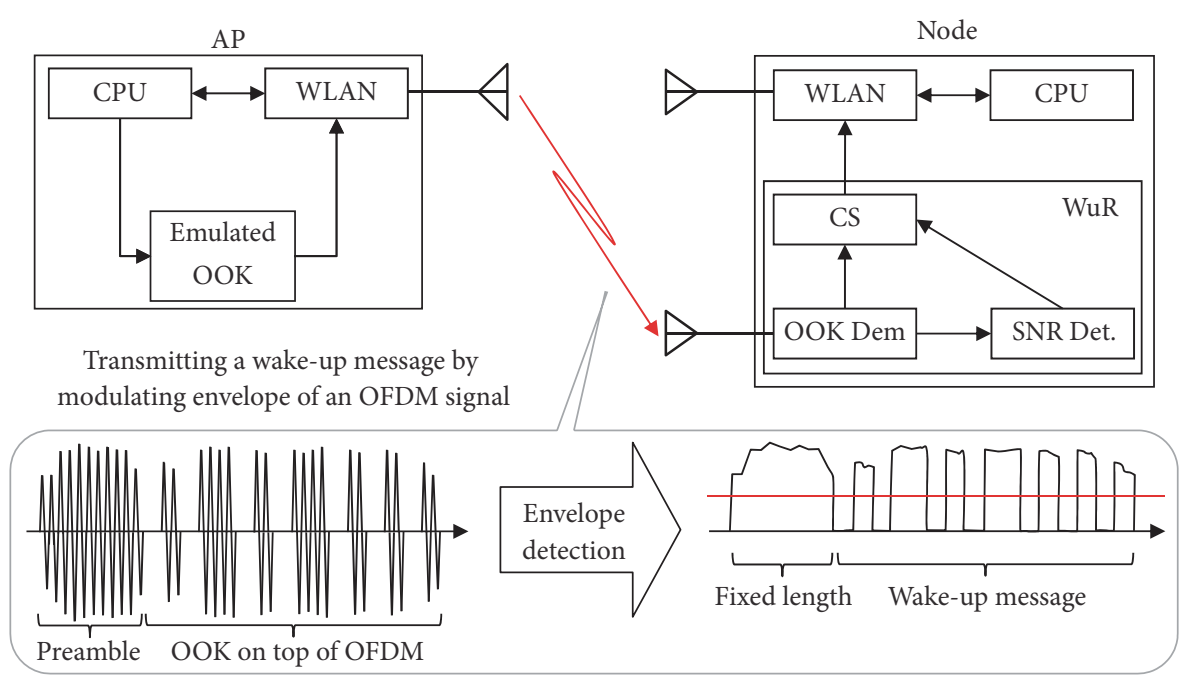

Figure 1: Framework for remote wake-up control of a mobile node. An AP transmits a wake-up message with a traffic indication map by applying the OOK modulation on the envelope of an OFDM signal. A node is equipped with a low-power WuR, which conducts envelope detection to receive a wake-up message, detects SNR, and performs carrier sense.

colocated WLAN module. If multiple nodes wake up simultaneously, their contention may cause collisions. To reduce collisions, only WuRs with high SNR above the threshold and packets ready will participate in this contention. Due to the wake-up latency, the channel is continuously idle in the wakeup period of a node that has just won the channel contention. As a result, other WuRs sense the channel as idle and may falsely activate their colocated WLAN modules [20]. Setting a SNR threshold helps to reduce false wake-up as well. It is desired that by proper control exactly one node with high SNR is activated so as to avoid false wake-up and collision and improve transmission rate.

Here, normalized SNR [18] instead of absolute SNR is used, and it is defined as the ratio of instantaneous SNR of a node to its average. Using normalized SNR helps to ensure proportional fair scheduling among nodes with different link qualities in a long term. But using a fixed SNR threshold may prevent a node in deep fading from accessing the channel in a short term. To alleviate this problem, the SNR threshold is adapted to the number of contending nodes.

The number of contending nodes with packets ready to be received changes with time, so does the SNR threshold. The AP continues transmitting all packets to the same node in a burst mode, where frames are spaced by SIFS (Short Interframe Space). The switch between nodes is indicated by a space of DIFS (DCF Interframe Space, longer than SIFS). By checking this DIFS, the number of contending nodes can be updated. Later, in Section 3, optimal SNR threshold is obtained from the latest number of contending nodes. In this way, the SNR threshold decreases with the number of contending nodes, which helps to alleviate the short-term fairness problem.

As link quality changes dynamically, the backoff counter is not resumed after each contention, but initialized per contention round using a fixed contention window $(\mathrm{CW})$.
2.2. Main Procedure. The main procedure of the proposed method is described as follows:

(1) An AP periodically broadcasts a WuM by applying OOK modulation on the envelope of WLAN signals, carrying a TIM indicating the availability of packets.

(2) A WuR measures RSSI/SNR on receiving a WuM, gets TIM from this WuM, and finds the number of contending nodes. Then, based on the number of contending nodes, each WuR determines its SNR threshold (based on a table).

(3) If there is a packet ready and its normalized SNR is greater than the threshold, a WuR initiates its backoff counter by a random value drawn from a CW and decreases its backoff counter per idle slot. A WuR immediately wakes up its WLAN module when its backoff counter reaches 0 .

(4) An activated WLAN module performs carrier sense again. If the channel remains idle, a WLAN module transmits a CTS to initiate its receiving from the AP. Otherwise, the channel is already busy, and this is a false wake-up, and the WLAN module goes to sleep again.

(5) On receiving a CTS frame from a node, the AP transmits all packets to the node in a burst, with a SIFS between adjacent frames, and notifies the end of this burst by clearing the More Data flag.

(6) When the transmission to a node finishes, there will be a space of DIFS, after which other nodes will contend to initiate their receiving from the AP. A space of DIFS or longer indicates the decrease by 1 in the number of contending nodes, and the SNR threshold is updated. Then, the procedures from step (3) are repeated. 


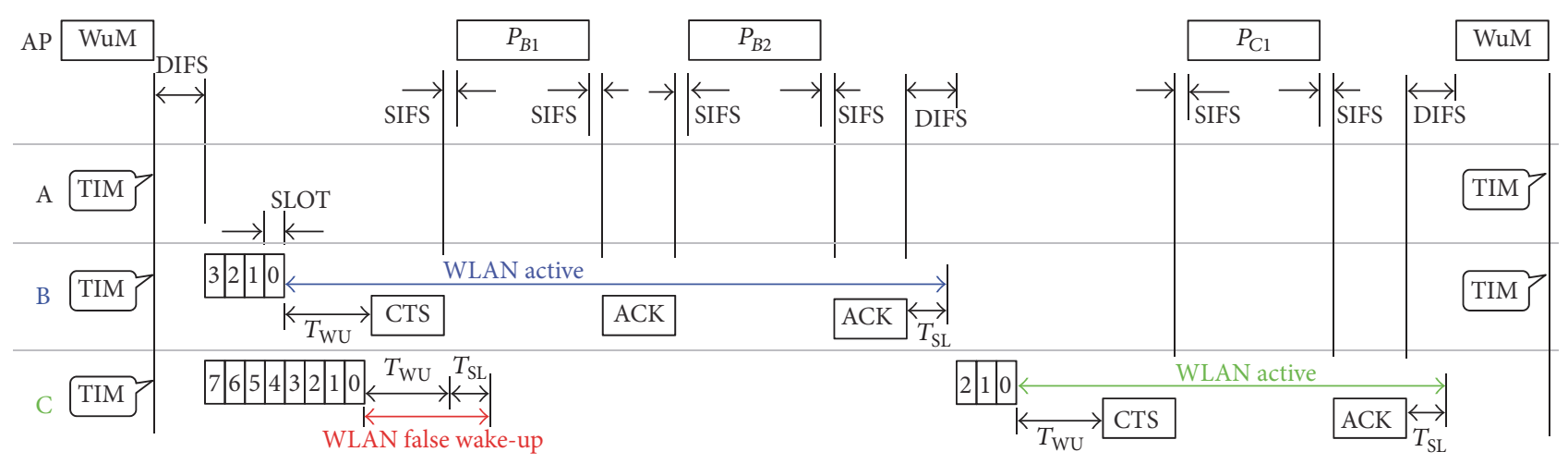

FIGURE 2: Wake-up control for the downlink transmission scheduling in a WLAN with an AP and 3 nodes. The AP broadcasts a WuM, which carries a TIM. The WuR of each node, on receiving a TIM and detecting its normalized SNR above the threshold, performs carrier sense, contending to activate its WLAN module. An activated WLAN module initiates the receiving from the AP. Packets to the same nodes are spaced by SIFS, and packets to different nodes are spaced by DIFS and random backoff ( $T_{\mathrm{WU}}=5$ slots $)$.

Occasionally, SNR of all remaining nodes (that have not received their packets yet) happen to be below the specified threshold. After DIFS, no nodes transmit and the channel remains idle, until the AP rebroadcasts a new WuM, which recovers the contention among the nodes.

A collision occurs when multiple nodes simultaneously transmit CTS. In this case, the AP, failing to receive an expected CTS, transmits a new WuM to resynchronize all the nodes.

A simple example is shown in Figure 2, where a WLAN consists of an AP and 3 nodes. At first the AP sends a WuM, indicating that there are packets ready for $A, B$, and $C$. In the first contention round, normalized SNR of $B$ and $C$ is above the threshold, and WuRs $B$ and $C$ start to compete for the channel. WuR $B$ happens to initiate its backoff counter with a small value of 3 and wins the contention. Its WLAN module is activated to send a CTS frame to initiate the receiving of $P_{B 1}$ and $P_{B 2}$ from the AP. Meanwhile, in the wake-up period (with a length of $T_{\mathrm{WU}}$ ) of $B$, the channel is idle, and WuR $C$ decreases its backoff counter. It happens that WLAN module of $C$ is also activated, which is a false wake-up. To reduce power consumption, a falsely activated node is put into the sleep mode again, and the probability of false wakeup will be controlled by adjusting the SNR threshold. A DIFS space indicates that the previous node $(B)$ has finished its receiving, and the number of nodes is reduced by one. Then, channel access is repeated, based on the latest SNR threshold corresponding to the number of remaining nodes that have not received their packets yet.

\section{Performance Analysis}

Here, we analyze the performance of the proposed method to deduce the optimal parameter. In the analysis, we use the following notations: $N$ is the CW value, $\gamma$ is normalized SNR with a PDF (probability density function) $f(\gamma)$ and a CDF (cumulative distribution function) $F(\gamma)$, and $\gamma_{0}$ is SNR threshold. In the case of Rayleigh fading, if instantaneous
TABLE 1: Main notations for the analysis.

\begin{tabular}{ll}
\hline$t_{\mathrm{DIFS}}$ & Time length of a DIFS \\
$t_{\mathrm{SIFS}}$ & Time length of an SIFS \\
$t_{\mathrm{EIFS}}$ & Time length of an EIFS \\
$t_{\mathrm{WuM}}$ & Time length of a wake-up message \\
$t_{\mathrm{CTS}}$ & Time length of a CTS frame \\
$t_{\text {Data }}$ & Time length of a DATA frame \\
$t_{\mathrm{ACK}}$ & Time length of an ACK frame \\
\hline$E_{T}$ & Power consumption at transmission state (1 watt) \\
$E_{R}$ & Power consumption at receiving state (1 watt) \\
$E_{I}$ & Power consumption at idle state (1 watt) \\
$E_{\mathrm{WR}}$ & Power consumption of WuR (1 mill-watt) \\
\hline$T_{S}$ & Time length of a contention slot \\
$N$ & Amount of slots in a contention window \\
$N_{\mathrm{WU}}$ & Amount of slots corresponding to wake-up period \\
$N_{\mathrm{SL}}$ & Amount of slots corresponding to sleep period \\
$m$ & Number of nodes participating in the contention \\
$M$ & Number of nodes with packets ready \\
$C_{m}^{M}$ & Number of combinations choosing $m$ out of $M$ elements \\
\hline
\end{tabular}

absolute SNR of a node $(i=1,2, \ldots, M)$ is $\gamma_{i}^{\prime}$ and its average value is $\bar{\gamma}_{i}^{\prime}$, its normalized $S N R, \gamma_{i}$, is defined as $\gamma_{i}=\gamma_{i}^{\prime} / \bar{\gamma}_{i}^{\prime}$, and $f(\gamma)=\exp (-\gamma)$ and $F(\gamma)=1-\exp (-\gamma)$ are the same for all nodes.

The length of wake-up period, $T_{\mathrm{WU}}$, varies with devices and mainly depends on the performance of high frequency oscillator. According to [24], it takes $139 \mu$ s to switch from $1 / 4$ clock rate to full clock rate and takes about $200 \mu$ s to generate a stable carrier frequency within $50 \mathrm{kHz}$ of the desired value in MAX7032 (https://www.maximintegrated.com/en/ products/comms/wireless-rf/MAX7032.html/). In the analysis, the wake-up latency is assumed to be $T_{\mathrm{WU}}=200 \mu \mathrm{s}$, approximately $N_{\mathrm{WU}}=22$ slots with $T_{S}=9 \mu \mathrm{s}$. Table 1 lists the main notations. 


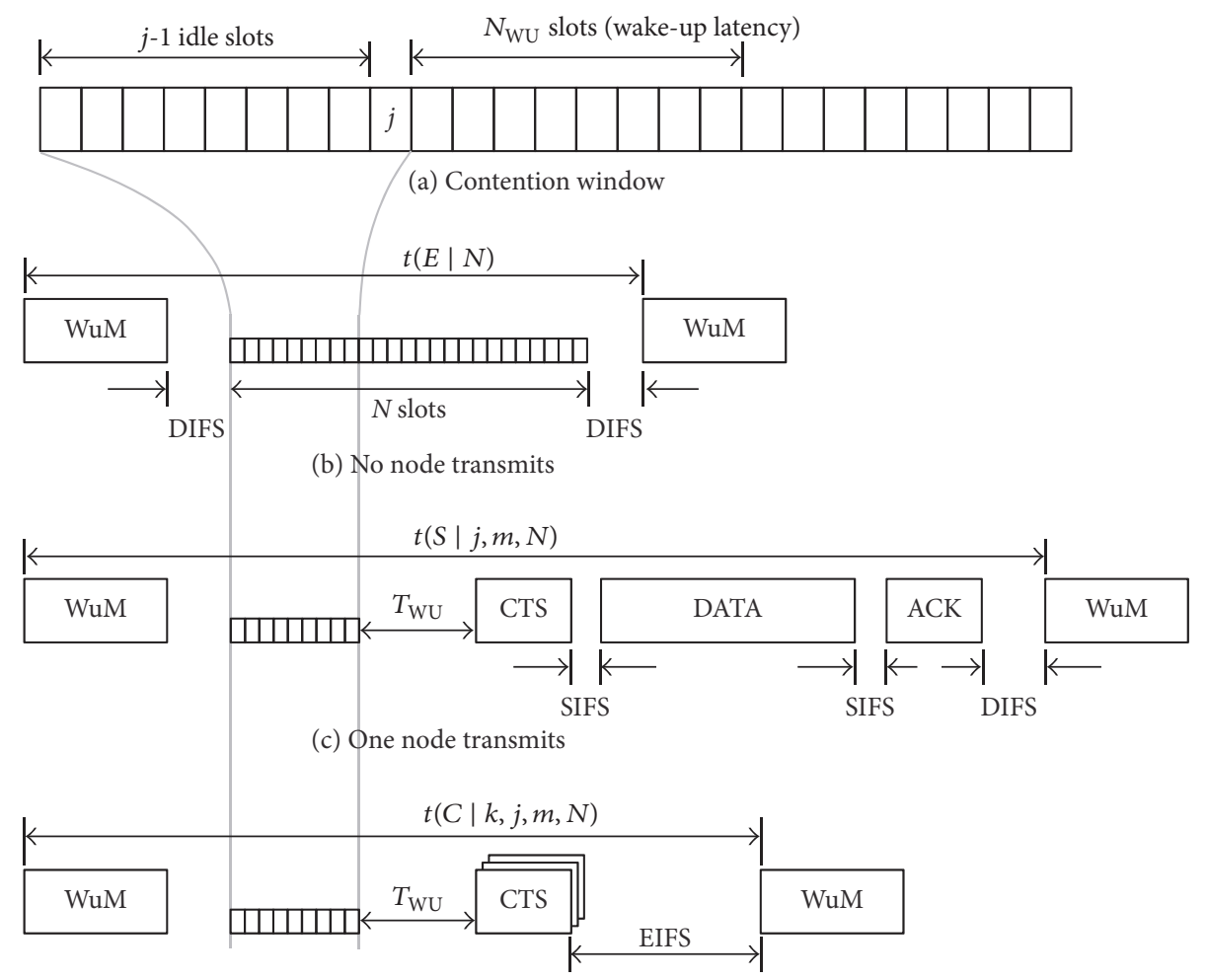

(d) A collision occurs due to concurrent transmissions

Figure 3: Different cases of a transmission. (a) A contention window with $N$ slots. (b) No node transmits, and the channel remains idle during the whole contention window. (c) Exactly one node transmits without a collision. (d) A collision occurs because multiple nodes simultaneously transmit.

3.1. Basic Analysis of Slotted Contention. Among all nodes, there are $M$ nodes with packets ready ( $M$ changes with time). Out of these $M$ nodes, with a probability

$$
P\left(m \mid \gamma_{0}\right)=C_{m}^{M} \cdot F\left(\gamma_{0}\right)^{M-m} \cdot\left[1-F\left(\gamma_{0}\right)\right]^{m},
$$

the normalized SNR of $m$ nodes is above the threshold $\gamma_{0}$.

Consider the $j$ th slot of the contention window, where multiple WLAN modules may be simultaneously activated because their backoff counters are first decreased to 0 at that slot. It should be noted that any WuR, whose backoff counter reaches 0 in the $N_{\mathrm{WU}}$ slots immediately after the $j$ th slot, also falsely activates its WLAN module, because no node transmits in this wake-up period. Falsely activated nodes are put into sleep again.

There are three possible cases after each channel contention.

(i) All nodes have a normalized SNR below $\gamma_{0}$ and no node transmits at all (Figure 3(b), denoted as E), which has a probability

$$
P\left(E \mid \gamma_{0}\right)=P\left(0 \mid \gamma_{0}\right)
$$

and the channel is idle for a time

$$
\begin{aligned}
t(E \mid N) & =t_{\mathrm{WuM}}+t_{\mathrm{DIFS}}+N \cdot T_{S}+t_{\mathrm{DIFS}}, \\
t\left(E \mid \gamma_{0}, N\right) & =t(E \mid N) \cdot P\left(E \mid \gamma_{0}\right) .
\end{aligned}
$$

Then, the AP rebroadcasts a WuM to start the next contention period.

(ii) One node transmits without any collision (Figure 3(c), denoted as $S$ ). This happens if exactly one node chooses the least timer value $j$, which has a probability

$$
\begin{aligned}
& P(S \mid j, m, N) \\
& \quad= \begin{cases}\frac{1}{N}, & 1 \leq j \leq N, m=1, \\
C_{1}^{m} \cdot \frac{(N-j)^{m-1}}{N^{m}}, & 1 \leq j \leq N-1, m \geq 2,\end{cases}
\end{aligned}
$$

and the overall success probability is

$$
P\left(S \mid \gamma_{0}, N\right)=\sum_{m=1}^{M} \sum_{j=1}^{N} P(S \mid j, m, N) P\left(m \mid \gamma_{0}\right) \text {. }
$$

Each successful transmission consumes the time

$$
\begin{aligned}
t(S \mid j, m, N)= & t_{\mathrm{WuM}}+t_{\mathrm{DIFS}}+j \cdot T_{S}+T_{\mathrm{WU}}+t_{\mathrm{CTS}} \\
& +t_{\mathrm{SIFS}}+t_{\mathrm{Data}}+t_{\mathrm{SIFS}}+t_{\mathrm{ACK}}+t_{\mathrm{DIFS}},
\end{aligned}
$$

and its average is equal to

$$
\begin{aligned}
& t\left(S \mid \gamma_{0}, N\right) \\
& \quad=\sum_{m=1}^{M} \sum_{j=1}^{N} t(S \mid j, m, N) P(S \mid j, m, N) P\left(m \mid \gamma_{0}\right) .
\end{aligned}
$$


And the number of successful transmissions per contention round is equal to

$$
L\left(S \mid \gamma_{0}, N\right)=1 \cdot \frac{P\left(S \mid \gamma_{0}, N\right)}{1-P\left(E \mid \gamma_{0}\right)}
$$

Of the other $m-1$ nodes whose backoff counters reach 0 in the remaining $N-j$ slots, the ones that reach 0 in the subsequent $N_{\mathrm{WU}}$ slots will experience false wake-up. This is equivalent to a binomial distribution [26] with $p=$ $N_{\mathrm{WU}} /(N-j)$ in the $m-1$ trials. Therefore, the average number of nodes that will experience false wake-ups is equal to

$$
\begin{aligned}
& L_{F}(S \mid j, m, N) \\
& = \begin{cases}(m-1) \frac{N_{\mathrm{WU}}}{N-j}, & 1 \leq j \leq N-N_{\mathrm{WU}}, m \geq 2, \\
(m-1), & N-N_{\mathrm{WU}}<j \leq N-1, m \geq 2 .\end{cases}
\end{aligned}
$$

The number of false wake-ups under a successful transmission is equal to

$$
\begin{aligned}
& L_{F}\left(S \mid \gamma_{0}, N\right)=\sum_{m=1}^{M} \frac{L_{F}(S \mid m, N) P\left(m \mid \gamma_{0}\right)}{1-P\left(E \mid \gamma_{0}\right)}, \\
& L_{F}(S \mid m, N)=\sum_{j=1}^{N} P(S \mid j, m, N) \cdot L_{F}(S \mid j, m, N) .
\end{aligned}
$$

(iii) Two or more nodes transmit simultaneously, which leads to a collision (Figure 3(d), denoted as C). A collision happens when $k \geq 2$ nodes choose the same least timer value $j$, which has a probability

$$
\begin{aligned}
& P(C \mid k, j, m, N) \\
& \quad= \begin{cases}C_{k}^{m} \cdot \frac{(N-j)^{m-k}}{N^{m}}, & 1 \leq j \leq N-1, m \geq 2, \\
\frac{1}{N^{m}}, & k=m, j=N, m \geq 2 .\end{cases}
\end{aligned}
$$

A collision leads to the channel waste for a time

$$
\begin{aligned}
t(C \mid k, j, m, N)= & t_{\mathrm{WuM}}+t_{\mathrm{DIFS}}+j \cdot T_{S}+T_{\mathrm{WU}} \\
& +t_{\mathrm{CTS}}+t_{\mathrm{EIFS}},
\end{aligned}
$$

and its average is equal to

$$
\begin{gathered}
t\left(C \mid \gamma_{0}, N\right)=\sum_{m=2}^{M} \sum_{j=1}^{N} \sum_{k=2}^{m} t(C \mid k, j, m, N) \\
\cdot P(C \mid k, j, m, N) P\left(m \mid \gamma_{0}\right) .
\end{gathered}
$$

The number of nodes simultaneously transmitting in a collision is equal to

$$
L(C \mid k, j, m, N)= \begin{cases}k, & 1 \leq j \leq N-1, m \geq 2, \\ m, & j=N, m \geq 2\end{cases}
$$

and its average is equal to

$$
\begin{aligned}
& L\left(C \mid \gamma_{0}, N\right)=\sum_{m=2}^{M} \frac{L(C \mid m, N) P\left(m \mid \gamma_{0}\right)}{1-P\left(E \mid \gamma_{0}\right)}, \\
& L(C \mid m, N) \\
& \quad=\sum_{j=1}^{N} \sum_{k=2}^{m} P(C \mid k, j, m, N) \cdot L(C \mid k, j, m, N) .
\end{aligned}
$$

Of the other $m-k$ nodes whose backoff counters reach 0 in the remaining $N-j$ slots, the ones that reach 0 in the subsequent $N_{\text {WU }}$ slots will experience false wake-up. This is equivalent to a binomial distribution with $p=N_{\mathrm{WU}} /(N-j)$ in the $m-k$ trials. Therefore, the average number of nodes that will experience false wake-ups is equal to

$$
\begin{aligned}
& L_{F}(C \mid k, j, m, N) \\
& \quad= \begin{cases}(m-k) \cdot \frac{N_{\mathrm{WU}}}{N-j}, & 1 \leq j \leq N-N_{\mathrm{WU}}, m \geq 2, \\
(m-k), & N-N_{\mathrm{WU}}<j<N, m \geq 2 .\end{cases}
\end{aligned}
$$

The average number of false wake-ups under a collision is equal to

$$
\begin{aligned}
& L_{F}\left(C \mid \gamma_{0}, N\right)=\sum_{m=2}^{M} \frac{L_{F}(C \mid m, N) P\left(m \mid \gamma_{0}\right)}{1-P\left(E \mid \gamma_{0}\right)}, \\
& L_{F}(C \mid m, N) \\
& \quad=\sum_{j=1}^{N} \sum_{k=2}^{m} P(C \mid k, j, m, N) \cdot L_{F}(C \mid k, j, m, N) .
\end{aligned}
$$

3.2. Optimal Parameter. The average time for each round is the weighted sum of three parts (idle waiting, successful transmission, and collision) as follows:

$$
\begin{aligned}
t\left(\gamma_{0}, N\right)= & t\left(E \mid \gamma_{0}, N\right)+t\left(S \mid \gamma_{0}, N\right) \\
& +t\left(C \mid \gamma_{0}, N\right)
\end{aligned}
$$

The average transmission rate is an average over all nodes. The transmission rate ( $r_{i}$ for the $i$ th node) is estimated from the absolute SNR $\left(\gamma \cdot \bar{\gamma}_{i}^{\prime}\right)$, based on the Shannon capacity, and the operator $E(\cdot)$ represents the computation of expectation.

$$
\begin{aligned}
& E\left(r \mid \gamma \geq \gamma_{0}\right)=\frac{1}{M} \sum_{i=1}^{M} E\left(r_{i} \mid \gamma_{i} \geq \gamma_{0}\right), \\
& E\left(r_{i} \mid \gamma_{i} \geq \gamma_{0}\right)=\frac{\int_{\gamma_{0}}^{\infty} \log _{2}\left(1+\gamma \cdot \bar{\gamma}_{i}^{\prime}\right) \cdot f_{\gamma_{i}}(\gamma) d \gamma}{\int_{\gamma_{0}}^{\infty} f_{\gamma_{i}}(\gamma) d \gamma} .
\end{aligned}
$$




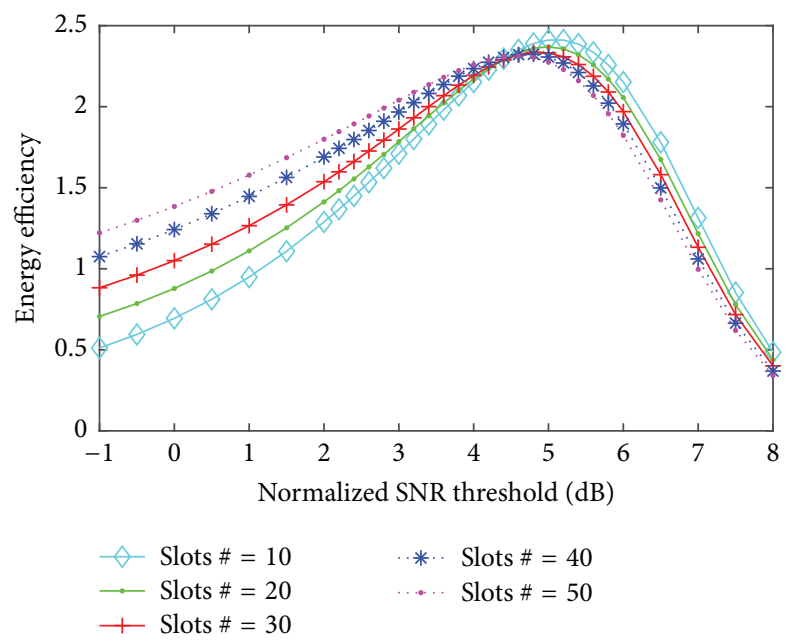

(a) Energy efficiency $\eta^{\prime}\left(\gamma_{0}, N\right)$

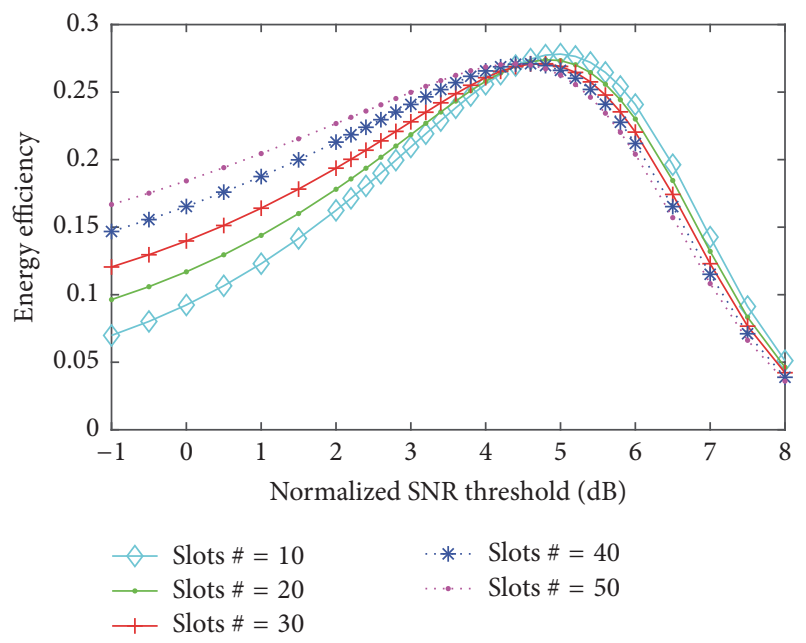

(b) Energy efficiency $\eta\left(\gamma_{0}, N\right)$

FIGURE 4: Different definitions of energy efficiency (average absolute SNR is equal to $20 \mathrm{~dB}$ ).

The average energy per round $\varepsilon\left(\gamma_{0}, N\right)$ is a sum of three parts: $\varepsilon_{S}\left(\gamma_{0}, N\right)$ for successful transmission, $\varepsilon_{C}\left(\gamma_{0}, N\right)$ for collisions, and $\varepsilon_{F}\left(\gamma_{0}, N\right)$ for false wake-ups as follows:

$$
\begin{aligned}
& \varepsilon\left(\gamma_{0}, N\right)=\varepsilon_{S}\left(\gamma_{0}, N\right)+\varepsilon_{C}\left(\gamma_{0}, N\right)+\varepsilon_{F}\left(\gamma_{0}, N\right), \\
& \varepsilon_{S}\left(\gamma_{0}, N\right)=\left(\left(T_{\mathrm{WU}}+T_{\mathrm{SL}}+2 \cdot t_{\mathrm{SIFS}}\right) \cdot E_{I}\right) \\
& \cdot L\left(S \mid \gamma_{0}, N\right) \\
&+\left(\left(t_{\mathrm{CTS}}+t_{\mathrm{ACK}}\right) \cdot E_{T}+t_{\mathrm{Data}} \cdot E_{R}\right) \\
& \cdot L\left(S \mid \gamma_{0}, N\right), \\
& \varepsilon_{C}\left(\gamma_{0}, N\right)=\left(\left(T_{\mathrm{WU}}+T_{\mathrm{SL}}+t_{\mathrm{EIFS}}\right) \cdot E_{I}+t_{\mathrm{CTS}} \cdot E_{T}\right) \\
& \cdot L\left(C \mid \gamma_{0}, N\right), \\
& \varepsilon_{F}\left(\gamma_{0}, N\right)=\left(T_{\mathrm{WU}}+T_{\mathrm{SL}}\right) \cdot E_{I} \\
& \cdot\left(L_{F}\left(S \mid \gamma_{0}, N\right)+L_{F}\left(C \mid \gamma_{0}, N\right)\right),
\end{aligned}
$$

where $\varepsilon_{S}\left(\gamma_{0}, N\right)$ is computed from $L\left(S \mid \gamma_{0}, N\right)$, the average number of successful transmissions, $\varepsilon_{C}\left(\gamma_{0}, N\right)$ is computed from $L\left(C \mid \gamma_{0}, N\right)$, the average number of nodes involved in a collision, and $\varepsilon_{F}\left(\gamma_{0}, N\right)$ is computed from $L_{F}\left(S \mid \gamma_{0}, N\right)+$ $L_{F}\left(C \mid \gamma_{0}, N\right)$, the average number of nodes experiencing false wake-up.

First, energy efficiency, defined as the ratio of the number of overall bits to the product of average transmission time and average energy, is considered as follows:

$$
\eta^{\prime}\left(\gamma_{0}, N\right)=\frac{E\left(r \mid \gamma \geq \gamma_{0}\right) \cdot t_{\text {Data }} \cdot P\left(S \mid \gamma_{0}, N\right)}{t\left(\gamma_{0}, N\right) \cdot \varepsilon\left(\gamma_{0}, N\right)} .
$$

Figure 4(a) shows the result, which plots energy efficiency with respect to normalized SNR threshold $\left(\gamma_{0}\right)$. Energy efficiency, under different contention window sizes $(N)$, has almost the same peak value, which indicates that the maximal energy efficiency is not very sensitive to contention window size. A small contention window usually cannot well remove the false wake-up, in that case a large SNR threshold needs to be used, and the SNR range is narrow. In contrast, using a relatively large CW helps to remove collisions and false wakeups in part, and a smaller SNR threshold can be used instead.

A problem is that average transmission rate $E(r \mid \gamma \geq$ $\gamma_{0}$ ) depends on the absolute SNR per link, which is difficult to obtain in the distributed environment. We notice that SNR threshold helps to both improve transmission rate and remove false wake-up. By separately considering the two items $E\left(r \mid \gamma \geq \gamma_{0}\right)$ and $\varepsilon\left(\gamma_{0}, N\right)$ in (21), that is, keeping one and removing the other, we find that reducing false wake-up usually requires higher SNR threshold.

Therefore, we choose to remove $E\left(r \mid \gamma \geq \gamma_{0}\right)$ from (21) and use the following definition of energy efficiency:

$$
\eta\left(\gamma_{0}, N\right)=\frac{t_{\text {Data }} \cdot P\left(S \mid \gamma_{0}, N\right)}{t\left(\gamma_{0}, N\right) \cdot \varepsilon\left(\gamma_{0}, N\right)}
$$

Figure 4(b) shows the energy efficiency $\eta\left(\gamma_{0}, N\right)$, which has a similar trend as that in Figure 4(a). As will be explained later, a high SNR threshold may increase the probability that no nodes have SNR above the threshold due to limit of channel coherence time. Therefore, a low SNR threshold is preferred, and a relatively large value is chosen for contention window, which is fixed to $N=50$ when there are no less than 5 nodes. Then, (22) is used to compute the optimal SNR threshold.

\section{Performance Evaluation}

In the simulation evaluation, we compare the following 4 methods. (1) First is the PSM mode [1] without using WuR. A WLAN module periodically wakes up to receive TIM, and if there are any packets buffered at the AP, it stays awake in the whole beacon period. Later simulation results will show that in this method a WLAN module will consume much more power than in other methods due to the idle waiting. (2) Second is the adaptive PSM mode (PSMAdapt) [2] without 


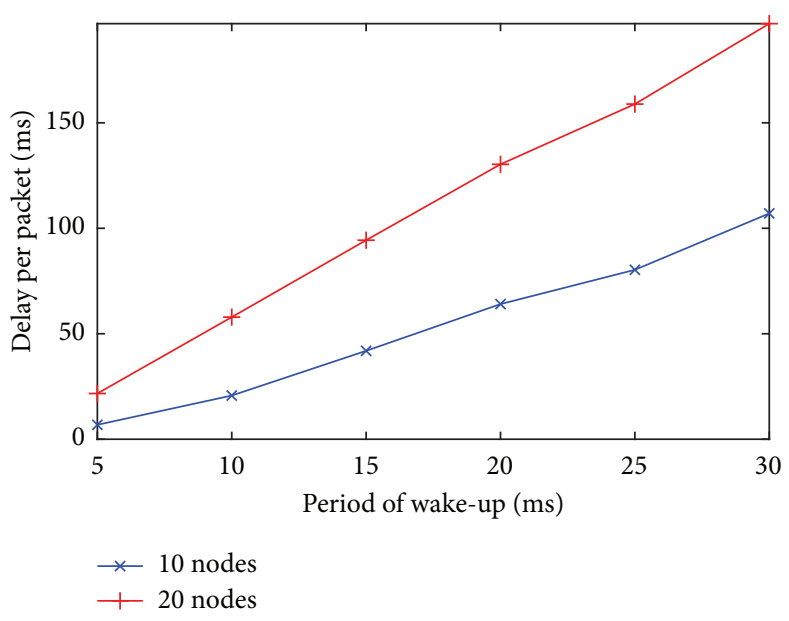

(a) Delay per packet (ms)

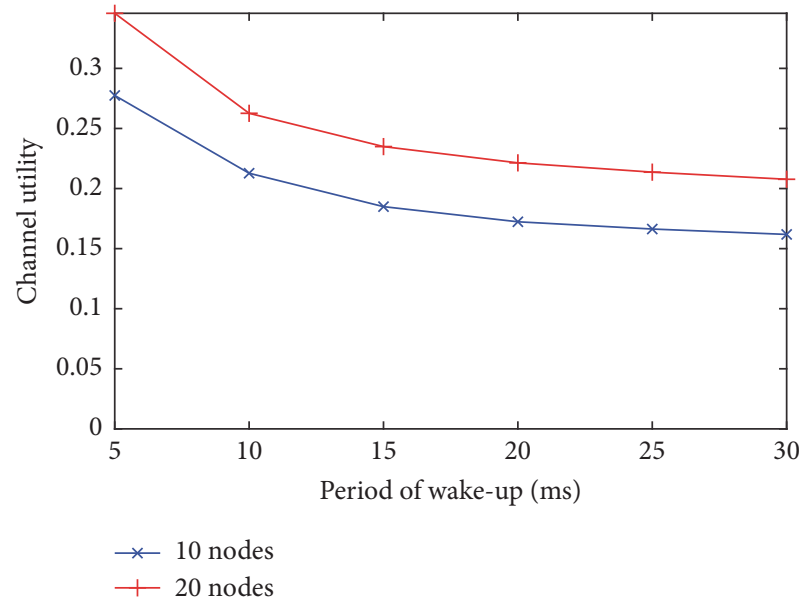

(b) Channel utilization

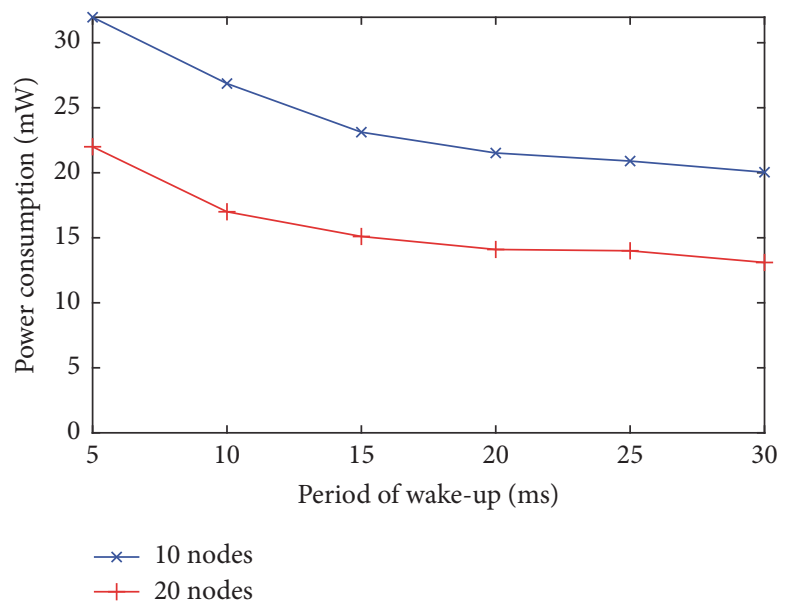

(c) Power consumption $(\mathrm{mW})$

FIGURE 5: Performance of WuRMUS under different WuM periods (traffic rate: 50 packets/s per node).

WuR. This is built on top of the PSM mode, but a node that has received all packets buffered at the AP will enter the sleep mode immediately. This helps to reduce most of the idle waiting in the PSM mode, although it causes large delay because packets which arrive after the node sleeps will not be received until the next TIM timing. (3) Third is the wake-up per node (WuR). The AP activates each node separately, without referring to their link quality. (4) Fourth is the wake-up with multiuser scheduling (WuRMUS), the proposed method.

We built a packet-level simulator in the MATLAB environment. We consider a WLAN with an AP and a variable number of nodes (average absolute SNR is equal to $20 \mathrm{~dB}$ ) and evaluate its performance with different traffic settings. Packet arrival follows the Poisson process. In the evaluation, we will consider four metrics: delay, channel utilization (the percentage of time that the channel is busy, including SIFS, DIFS, and backoff slots), power consumption per node, and throughput. The results are the average of 50 runs with different seeds. The transmission rate is decided by SNR [27]. IEEE 802.11a is adopted and packet length is 1000 bytes. Main parameters are shown in Table 2. In all methods, opportunistic packet aggregation [28] is used, so that multiple packets that arrived before the transmission timing are transmitted together if they can be aggregated (with the constraint that the transmission time will not be larger than the one for transmitting a packet at the lowest rate).

4.1. Effect of WuM Period. In the proposed method, the WuM period plays an important role. Here we evaluate the performance of the proposed method under different WuM periods, and the results are shown in Figure 5. Unsurprisingly, the delay per packet increases with the WuM period (Figure 5(a)). Figure 5(b) shows that channel utilization decreases as WuM period increases. This is because a large WuM period increases the opportunity of packet aggregation and improves channel efficiency. This will reduce the time that a node stays awake to receive its packets buffered at the AP. As a result, power consumption per node decreases as WuM period increases, as shown in Figure 5(c). Because a very small WuM period will increase the overhead and energy consumption 


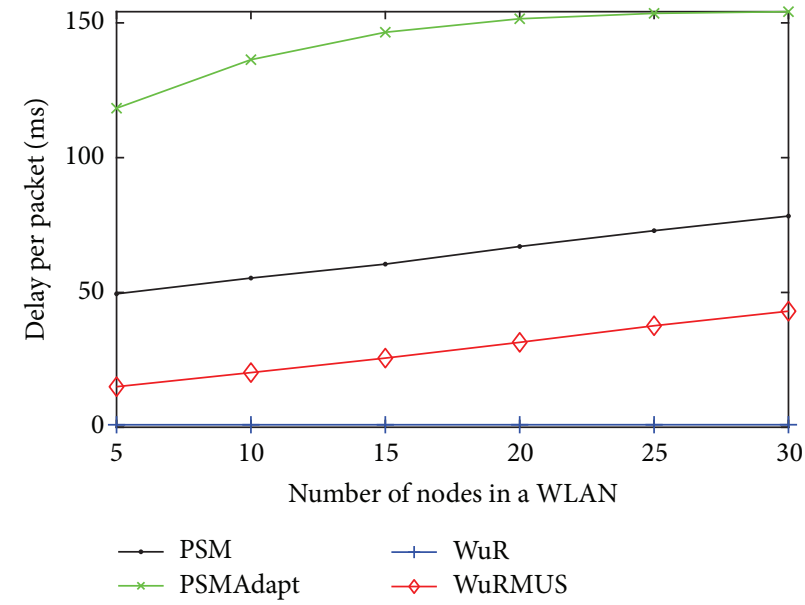

(a) Delay per packet (ms)

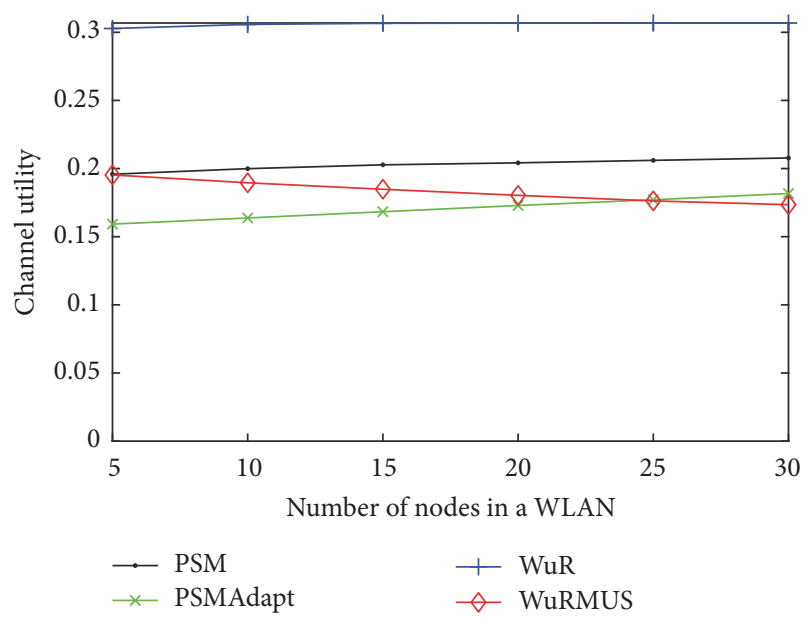

(b) Channel utilization

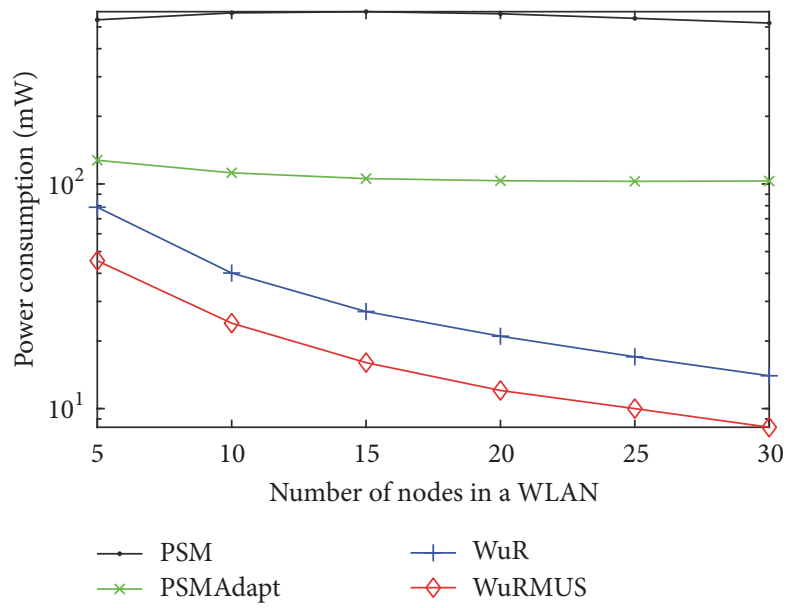

(c) Power consumption $(\mathrm{mW})$

Figure 6: Performance of different methods under different numbers of nodes (overall traffic rate: 500 packet/s).

TABLE 2: Main parameters for simulation.

\begin{tabular}{lc}
\hline PHY & IEEE 802.1la, propagation: free-space \& two-ray \\
MAC & CSMA, $T_{\text {SLOT }}=9 \mu s, T_{\text {SIFS }}=16 \mu s, T_{\text {DIFS }}=34 \mu \mathrm{s}$ \\
Period & Beacon: $100 \mathrm{~ms}$, TIM: $200 \mathrm{~ms}$, WuM: $10 \mathrm{~ms}$ \\
WuM & $0.4 \mathrm{~ms}$ in WuRMUS, $0.1 \mathrm{~ms}$ in WuR. \\
Packet & $L=1,000$ byte \\
Traffic & Packet arrival time follows Poisson process \\
Bit rate & Decided by SNR \\
CW & 50 slots \\
Latency & Wake-up latency: $22 \times T_{\text {SLOT }}$, sleep: $2 \times T_{\text {SLOT }}$ \\
Power & WLAN: receiving/idle $1 \mathrm{~W}$, transmission $1 \mathrm{~W}$; WuR: $1 \mathrm{~mW}$ \\
\hline
\end{tabular}

while a very large WuM period will increase the delay, in the following, the $\mathrm{WuM}$ period is chosen to be $10 \mathrm{~ms}$.

4.2. Under Different Numbers of Nodes. Here we evaluate the performance of different methods with respect to the number of nodes in a WLAN. In this evaluation, the overall traffic is fixed to 500 packet/s and the amount of traffic per node decreases as the number of nodes increases. Because packets to different nodes arrive at different timing, the number of nodes that have packets buffered at the AP changes with time.

Figure 6(a) shows the delay per packet in different methods. WuRMUS lies between PSM and WuR, and PSMAdapt has the largest delay. In PSM, each node wakes up per TIM timing (period $=200 \mathrm{~ms}$ ) and shifts to the sleep state if no packet is available. Any packets arriving immediately after a node sleeps will have a large delay. As for PSMAdapt, a node shifts to the sleep mode more aggressively, which leads to larger delay. In WuRMUS, the delay per packet increases with the number of nodes. As the number of nodes increases, more nodes have buffered packets and will participate in the transmission scheduling. Then, a higher SNR threshold will be selected. But the channel does not change much within a short time (channel coherence time), and the probability that no nodes have SNR above the specified SNR threshold increases, which increases the delay in WuRMUS. The delay per packet in WuR is almost always nearly 0 , at the cost of more overhead and power consumption. 


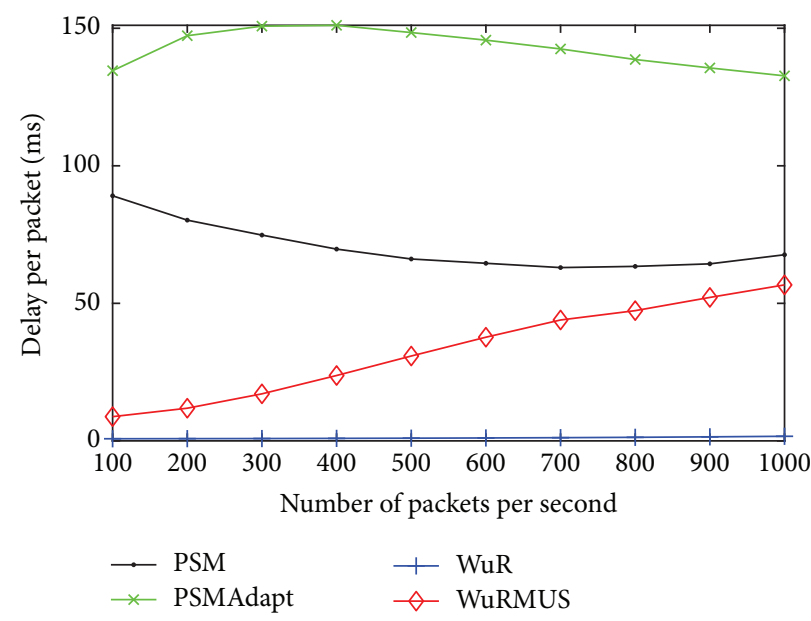

(a) Delay per packet (ms)

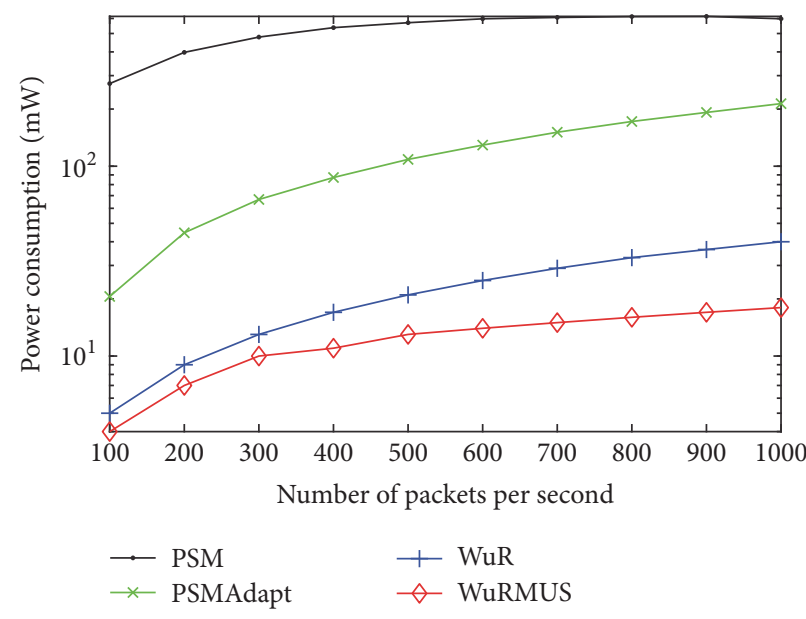

(b) Power consumption $(\mathrm{mW})$

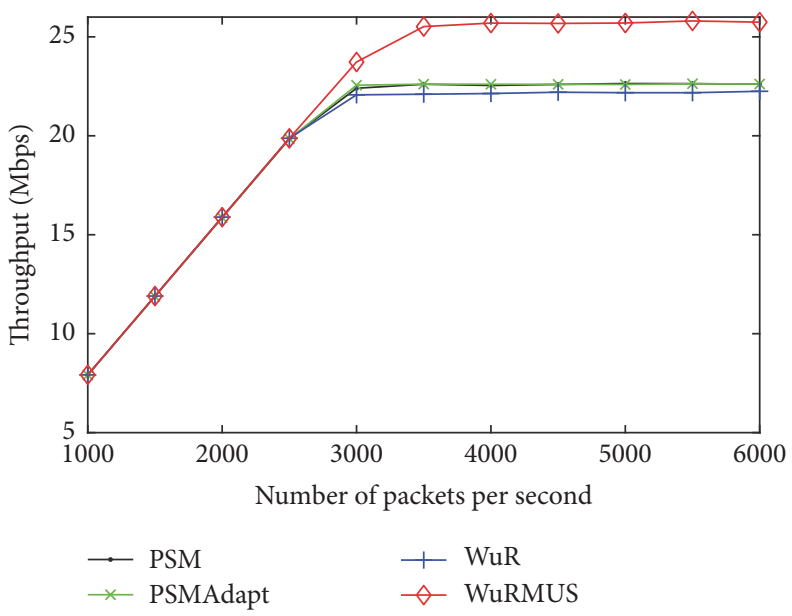

(c) System throughput (Mbps)

Figure 7: Performance of different methods under different volumes of traffic (number of nodes $=20$ ).

Figure 6(b) shows channel utilization in different methods. Channel utilization is the highest in WuR due to its transmission of WuM per node. PSMAdapt has lower channel utilization compared with PSM, because with more aggressive sleep policy, more packets are buffered at the TIM timing, which enables more chance of packet aggregation and reduces overhead. In WuRMUS, channel utilization decreases as the number of nodes increases, due to two reasons. (i) The SNR threshold increases with the number of nodes, which, at the cost of delay, increases the chance of packet aggregation. But WuRMUS has much smaller delay than PSM by using a smaller WuM period. (ii) With more nodes, the effect of multiuser diversity becomes more obvious and the transmission rate is improved.

Figure 6(c) shows power consumption per node. Unsurprisingly, power consumption is the highest in PSM, because each node, once detecting that there is a packet ready to be received according to the TIM included in a beacon, will wait until the next beacon announces that no packet remains. Using a more aggressive power-save mode in PSMAdapt effectively reduces the power consumption compared with
PSM, but power consumption is still nearly constant, because some nodes that failed to capture the channel first have to stay awake, waiting for other nodes to finish receiving. In WuR and WuRMUS, power consumption decreases as the number of nodes increases, this is because (i) the WLAN module of a node is active only when receiving a packet, and (ii) the overall traffic is fixed and the amount of traffic per node decreases, so does the active time per node. WuRMUS further decreases power consumption per node compared with WuR, and its gain ranges from $40 \%$ to $43 \%$. This is achieved by (i) improving transmission rate by setting an SNR threshold and (ii) increasing the opportunities of packet aggregation by periodically transmitting WuMs.

4.3. Under Different Traffic Volume. Next we fix the number of nodes to 20 and adjust the overall traffic to investigate the changes in delay, power consumption, and system throughput.

Figure 7(a) shows the delay per packet. As the amount of overall traffic increases, on average, the delay decreases in PSM. But the delay in WuRMUS has a different trend. 
Although the number of overall nodes is fixed, with more traffic, the number of nodes with buffered packets increases, so does the SNR threshold. Then, the probability that no nodes have SNR above the specified SNR threshold increases, which increases the delay in WuRMUS. But the delay in WuRMUS is still less than that in PSM.

Figure 7(b) shows that power consumption per node increases with the amount of traffic as well. Anyway, power consumption is always the lowest in WuRMUS. When there is little traffic ( $\leq 300 \mathrm{packet} / \mathrm{s})$, the superiority of WuRMUS over WuR is not very obvious. But with more traffic, WuRMUS reduces power consumption per node, by $35 \%$ to $55 \%$, compared with WuR.

Figure $7(\mathrm{c})$ shows that throughput increases with the amount of overall traffic and finally approaches constant values in all methods when the network saturates. Although WuR and WuRMUS have relatively large overhead in times of light traffic, in the saturation state, many packets are aggregated and the overhead of wake-up control is not very obvious. Therefore, in the saturation state, the throughput of WuR is only a little lower than that of PSM and PSMAdapt. WuRMUS achieves higher throughput than other methods because it exploits multiuser scheduling to improve transmission rates. It should be noted that WuRMUS does not realize throughput fairness among nodes. Instead, it ensures a proportional fairness, and interested readers may refer to [18] for more details.

In summary, WuRMUS reduces power consumption compared with other methods. In times of light traffic, WuRMUS also reduces delay compared with PSM and PSMAdapt and reduces channel utilization compared with WuR. In times of medium traffic or many nodes, the number of nodes that have buffered packets increases, which increases the SNR threshold. But the channel does not change completely randomly due to the limit of channel coherence time. Therefore, in WuRMUS, the probability that no nodes have SNR greater than specified SNR increases, which leads to large delay. How to solve this problem is left as a future work. In times of heavy traffic, the overhead of wake-up control in WuRMUS is not obvious after packet aggregation. Instead, applying multiuser scheduling helps to improve system throughput.

\section{Conclusion}

This paper studies the transmission scheduling in the downlink of a WLAN by using a wake-up radio to remotely activate a WLAN module to receive packets on demand. Instead of wake-up per node, we proposed a broadcastbased wake-up control framework to reduce the overhead and improve the transmission rate. This brings new issues like transmission collisions and false wake-up. These problems are solved by setting a SNR threshold. In this way, the proposed method reduces transmission collisions and false wake-ups and improves transmission rate. Simulation evaluations verify that the proposed method (i) effectively reduces power consumption of nodes compared with stateof-the-art methods, (ii) reduces delay compared with powersave mode in times of light traffic, and (iii) improves system throughput compared with other methods in the saturation state. In the future, we will also study how to further reduce the delay in the proposed method.

\section{Conflicts of Interest}

The authors declare that there are no conflicts of interest regarding the publication of this paper.

\section{References}

[1] "Wireless LAN Medium Access Control (MAC) and Physical Layer (PHY) Specification,” IEEE Std. 802.11, 2012.

[2] Y. He and R. Yuan, "A novel scheduled power saving mechanism for 802.11 wireless LANs," IEEE Transactions on Mobile Computing, vol. 8, no. 10, pp. 1368-1383, 2009.

[3] G. Anastasi, M. Conti, M. Di Francesco, and A. Passarella, "Energy conservation in wireless sensor networks: a survey," Ad Hoc Networks, vol. 7, no. 3, pp. 537-568, 2009.

[4] S. Tang, H. Yomo, Y. Kondo, and S. Obana, "Wake-up receiver for radio-on-demand wireless LANs," EURASIP Journal on Wireless Communications and Networking, vol. 2012, article no. 42, 2012

[5] J. Oller, I. Demirkol, J. Casademont, J. Paradells, G. Gamm, and L. Reindl, "Has time come to switch from duty-cycled MAC protocols to wake-up radio for wireless sensor networks?" IEEE/ACM Transactions on Networking, vol. 24, no. 2, pp. 674687, 2016.

[6] I. Demirkol, C. Ersoy, and E. Onur, "Wake-up receivers for wireless sensor networks: benefits and challenges," IEEE Wireless Communications Magazine, vol. 16, no. 4, pp. 88-96, 2009.

[7] V. Jelicic, M. Magno, D. Brunelli, V. Bilas, and L. Benini, "Analytic comparison of wake-up receivers for WSNs and benefits over the wake-on radio scheme," in Proceedings of the 7th ACM Workshop on Performance Monitoring and Measurement of Heterogeneous Wireless and Wired Networks (PM2HW2N '12), pp. 99-106, ACM, Paphos, Cyprus, October 2012.

[8] D. K. McCormick, "IEEE technology report on wake-up radio: an application, market, and technology impact analysis of lowpower/low-latency 802.11 wireless LAN interfaces," 802.11ba Battery Life Improvement: IEEE Technology Report on Wake-Up Radio, pp. 1-56, Nov 2017.

[9] S. C. Folea and G. Mois, "A low-power wireless sensor for online ambient monitoring," IEEE Sensors Journal, vol. 15, no. 2, pp. 742-749, 2015.

[10] D. Spenza, M. Magno, S. Basagni, L. Benini, M. Paoli, and C. Petrioli, "Beyond duty cycling: wake-up radio with selective awakenings for long-lived wireless sensing systems," in Proceedings of the IEEE Conference on Computer Communications (INFOCOM '15), pp. 522-530, Kowloon, Hong Kong, April 2015.

[11] F. Z. Djiroun and D. Djenouri, "MAC protocols with wake-up radio for wireless sensor networks: a review," IEEE Communications Surveys \& Tutorials, vol. 19, no. 1, pp. 587-618, 2017.

[12] H. Qin and W. Zhang, "ZigBee-assisted power saving management for mobile devices," IEEE Transactions on Mobile Computing, vol. 13, no. 12, pp. 2933-2947, 2014.

[13] J. Guo, Z. Song, Y. Cui, Z. Liu, and Y. Ji, "Energy-efficient resource allocation for multi-user mobile edge computing," in Proceedings of the IEEE Global Communications Conference (GLOBECOM), IEEE, 2017.

[14] Y. Cui, W. He, C. Ni, C. Guo, and Z. Liu, "Energy-efficient resource allocation for cache-assisted mobile edge computing," 
in Proceedings of the 2017 IEEE 42nd Conference on Local Computer Networks (LCN), pp. 640-648, Singapore, October 2017.

[15] X. Qin and R. A. Berry, "Distributed approaches for exploiting multiuser diversity in wireless networks," IEEE Transactions on Information Theory, vol. 52, no. 2, pp. 392-413, 2006.

[16] S. Adireddy and L. Tong, "Exploiting decentralized channel state information for random access," IEEE Transactions on Information Theory, vol. 51, no. 2, pp. 537-561, 2005.

[17] C.-S. Hwang and J. M. Cioffi, "Opportunistic CSMA/CA for achieving multi-user diversity in wireless LAN," IEEE Transactions on Wireless Communications, vol. 8, no. 6, pp. 2972-2982, 2009.

[18] S. Tang, "Distributed multiuser scheduling for improving throughput of wireless LAN," IEEE Transactions on Wireless Communications, vol. 13, no. 5, pp. 2770-2781, 2014.

[19] M. Park, S. Azizi, R. Stacey et al., "Proposal for wake-up receiver (WUR) study group," Tech. Rep., IEEE 802.11-16/0722r1, May 2016.

[20] S. Tang and S. Obana, "Tight integration of wake-up radio in wireless LANs and the impact of wake-up latency," in Proceedings of the 59th IEEE Global Communications Conference, GLOBECOM 2016, USA, December 2016.

[21] K. Chebrolu and A. Dhekne, "Esense: communication through energy sensing," in Proceedings of the 15th Annual ACM International Conference on Mobile Computing and Networking (MobiCom '09), pp. 85-96, ACM, Beijing, China, September 2009.

[22] Y. Kondo, H. Yomo, S. Tang et al., "Energy-efficient WLAN with on-demand AP wake-up using IEEE 802.11 frame length modulation," Computer Communications, vol. 35, no. 14, pp. 1725-1735, 2012.

[23] S. Tang, H. Yomo, and Y. Takeuchi, "Optimization of frame length modulation-based wake-up control for green WLANs," IEEE Transactions on Vehicular Technology, vol. 64, no. 2, pp. 768-780, 2015.

[24] X. Zhang and K. G. Shin, "E-mili: energy-minimizing idle listening in wireless networks," IEEE Transactions on Mobile Computing, vol. 11, no. 9, pp. 1441-1454, 2012.

[25] F. Talucci, M. Gerla, and L. Fratta, "MACA-BI (MACA by invitation)-a receiver oriented access protocol for wireless multihop networks," in Proceedings of the 1997 International Symposium on Personal, Indoor and Mobile Radio Communications, (PIMRC '97), pp. 435-439, September 1997.

[26] D. P. Bertsekas and J. N. Tsitsiklis, Introduction to Probability, Athena Scientific, Belmont, Mass, USA, 2002.

[27] G. Holland, N. Vaidya, and P. Bahl, "A rate-adaptive MAC protocol for multi-Hop wireless networks," in Proceedings of the 7th Annual International Conference on Mobile Computing and Networking (MobiCom '01), pp. 236-251, Rome, Italy, 2001.

[28] Y. Lin and V. W. S. Wong, "Frame aggregation and optimal frame size adaptation for IEEE 802.11n WLANs," in Proceedings of the IEEE Global Telecommunications Conference (GLOBECOM '06), pp. 1-6, San Francisco, Calif, USA, NovemberDecember 2006. 


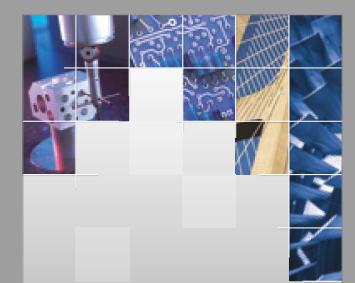

\section{Enfincering}
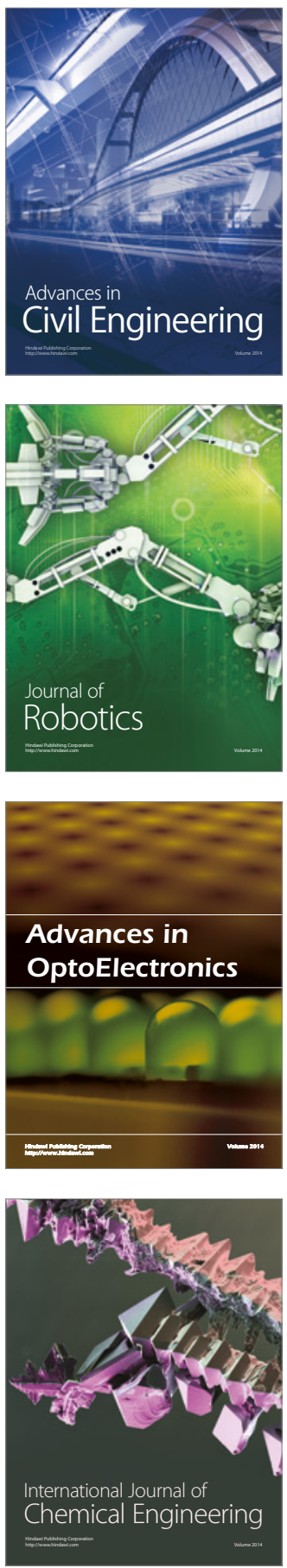

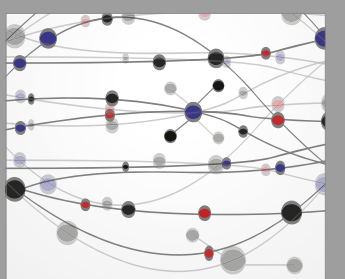

The Scientific World Journal

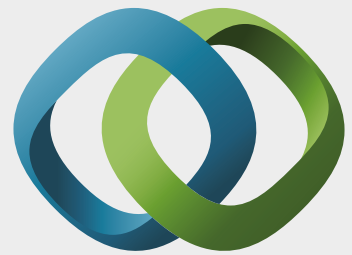

\section{Hindawi}

Submit your manuscripts at

https://www.hindawi.com
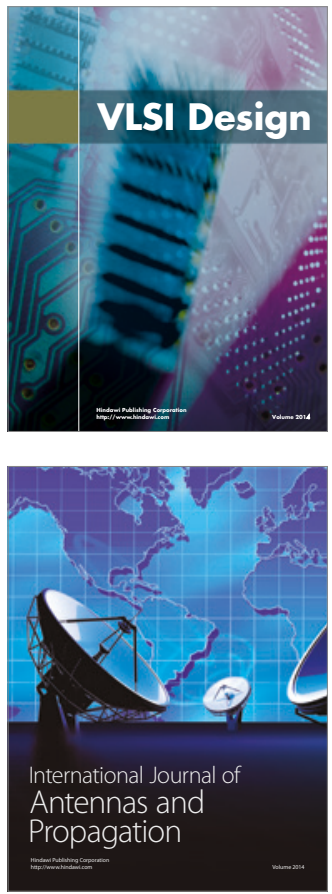

\section{Rotating}

Machinery
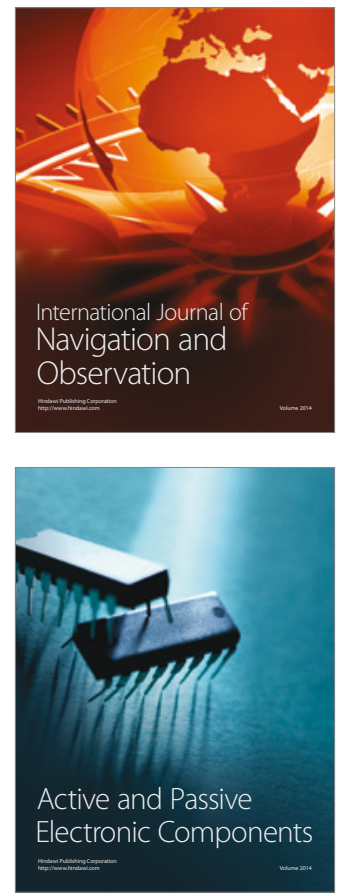
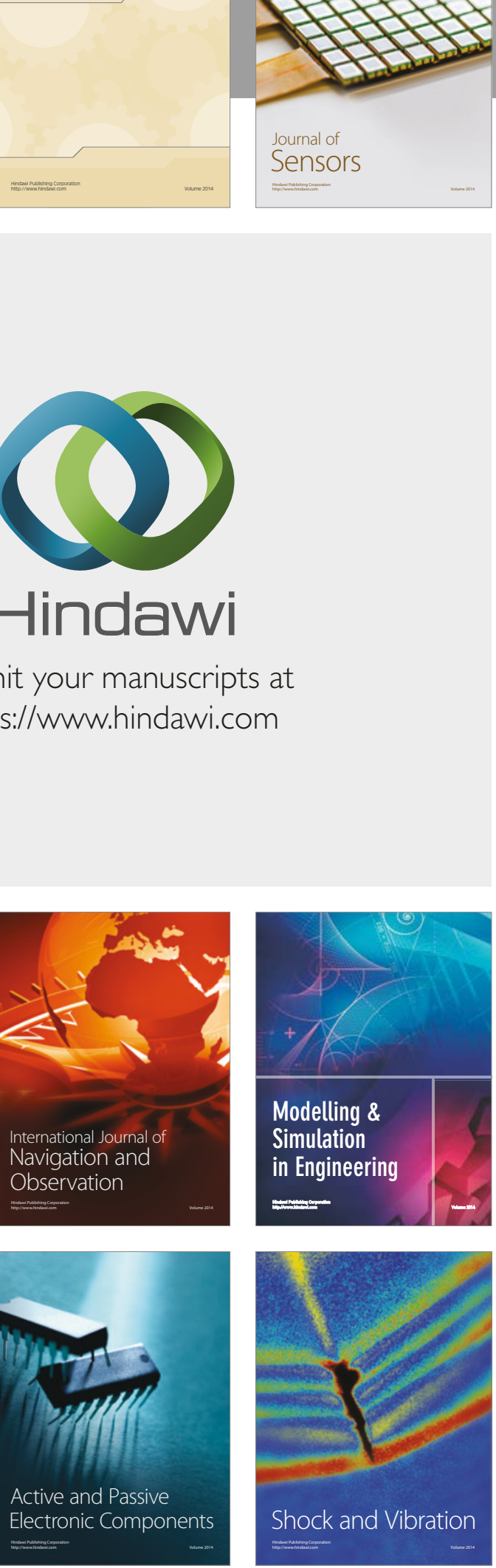
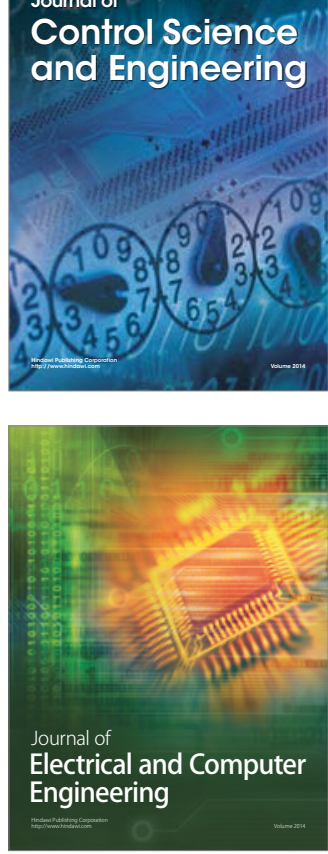

Distributed

Journal of

Control Science

and Engineering
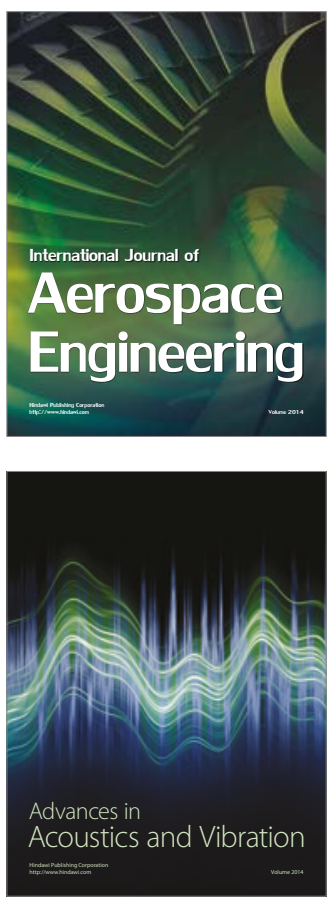

Sensor Networks 